\title{
Why should a neurologist worry about climate change?
}

Climate change is accepted by the majority of informed authorities as a clear feature of the Anthropocene era, and is becoming the lived experience shared by many of us globally, as we comment with increasing frequency about the unseasonal weather and the environment transforming around us. Whilst individual extreme events remain difficult to ascribe to a given cause, the sum total of such occurrences is beyond chance. Irrespective of personal perspectives, why as professionals should this concern us, and if it should, what, if anything, can we do?

Epilepsy can serve as an example. Epilepsy is a common global healthcare issue that disproportionately burdens those with less capacity to counter the difficulties it presents. Those living in poverty, or with limited or no availability of healthcare, and therefore limited access to antiepileptic drugs, are more likely to suffer from epilepsy, its consequences and comorbidities and die earlier as a result. Even in economically powerful nations, epilepsy is both more common in disadvantaged socioeconomic groups and is typically associated with poorer quality of life. People already deprived in these ways are likely to have less resilience to deal with the pervasive effects of climate change.

Moreover, for some people with epilepsy, climate change will interact directly with inescapable biology. For example, the rare severe epilepsy Dravet Syndrome is usually due to mutations in the neuronal sodium channel subunit gene SCN1A. The function of the encoded protein is exquisitely sensitive to ambient temperature, a phenomenon that may be exacerbated in mutant protein. Anecdotal evidence suggests that heatwaves are bad for people with Dravet Syndrome. During the recent high temperatures in Europe, parents reported to the UK support group that their children with Dravet Syndrome were experiencing more seizures and more lethargy. In Australia, a girl with Dravet Syndrome had been free of seizures with anti-epileptic drug treatment for two years: she went walking outside on the hottest day in Melbourne for years, with a peak of 46.5 degrees Celsius. She was found dead, with the suspicion that the extreme temperature was the cause. Many other genetic changes (for example pathogenic variants in the genes GABRG2, PCDH19, CHD2, STX1B) predispose people to epilepsy with initial or subsequent seizures often provoked by fever or, possibly, higher ambient temperatures, though the underlying mechanisms remain poorly understood. More and more genetic causes of the epilepsies are being identified, subdividing loose classical groupings into specific categories driven by mutation in one gene or a set of related genes. Exploration of pathophysiology in these epilepsies may help us explain increased vulnerability to seizures associated with higher temperatures, or other commonly-reported and ill-understood precipitants, such as stress or concurrent infection.

Climate change is leading to changes beyond temperature extremes. It may alter the severity or frequency of events such as heatwaves and floods, and the range of vectors of infections inducing fevers or epilepsy, all amongst the challenges overloading already-teetering economies, and adding to demands on well-resourced nations. These events will all have serious consequences for people with epilepsy. Poor sleep and anxiety can aggravate seizures in all epilepsies, even if seizures are otherwise well controlled: it must be difficult to sleep if you have lost your home to a landslide. The stability of medications may not be possible to guarantee with environmental fluctuations beyond designed tolerances. There are likely to be many other unforeseen downsides to climate change and the management of epilepsy.

Climate change has received little attention in neurology, and conversely neurologists have said little about the dangers of climate change for people with epilepsy or other neurological disorders. We have 
barely any information about the effects of climate change on fundamental biological parameters, from gene mutation rate, through to gene expression, brain network changes and the clinical manifestations of neurological disease. We can expect that this current era of climate change, with unpredictable environments, rapid changes in circumstances, and disruptions to supply chains will have distinct and major impacts on the practice of medicine. So, in our professional lives at least, we should put this on the agenda.

Action to remedy climate change requires concerted efforts. The Climate Coalition, UK) and special interest groups (e.g. UK Health Alliance on Climate Change) as well as locality-based action groups (e.g. Transition Town Totnes, Hook Norton Low Carbon). As a particular example, The Low Carbon Hub in Oxford, UK has carried out 38 solar panel installations on schools, businesses and community assets in Oxfordshire. These are funded by 808 community investors and save 1562 tonnes of carbon dioxide annually.

Individuals also need to make their own changes. Climate change is a critical new concern with which we, as clinicians, must engage. At the very least, we need to understand what may happen.

Perhaps there is more we can do ourselves. We provide one simple example. The authors themselves are neither eco-warriors nor carbon saints. In our professional endeavours, we too fly around the world. Our estimated total carbon emissions for a recent typical year's conference and meeting travel is 22.5 tonnes (see Figure), a sizeable additional contribution at the individual level. Air travel may be the biggest contribution to carbon emissions we make individually as neurologists. But conferences are important: there is formal learning, there are off-line meetings that drive science forward, and peer conversations that influence and improve practice around the world. Moreover, the value of face-to-face contact is undoubted. Such events also generate organisational income. We need to establish the cost effectiveness of such events, of their range, frequency distribution and value across the globe, and factor these into calculations of carbon cost and environmental impact. Air travel overall, though only accounting for a small fraction of human carbon emissions, is on the rise, and its environmental damage is difficult to engineer away. We estimated the carbon emissions of the (presumed air) travel of the approximately 3200 participants to one recent international epilepsy conference at 2000 tonnes of carbon dioxide. It is hard to count the number of international medical conferences worldwide, but one conference organiser estimated 1500 conferences annually. Many conferences are considerably bigger than a typical epilepsy conference, but even if they were all like our epilepsy conference, travel to these meetings alone would result in 3 million tonnes of carbon dioxide being emitted annually, which is equivalent to the total annual emissions of the entire country of Madagascar (population 25 million). Madagascar is the subject of the first World Bank report on climate change and health published this year, and is identified as being particularly vulnerable. Is this equitable? How can fairness in this context be calculated? We need more data, and opportunities for advances in technology to bring people together virtually, with the same warmth and social nuances of personal meetings (calculating the relative environmental costs of such technology also). None of this is beyond our wit, were we to have the will to try. In the interim, there are carbon offset programmes, but, to make a real difference, we should perhaps consider flying a little less.

Climate change is with us already, and, viewed from the perspective of attempting to continue to provide holistic healthcare, will affect both our patients and our professions at many levels. For all our sakes, and for the generations to come, we need to engage with what is happening, and consider our actions and their consequences. If there are simple steps we can all take that might make a difference - and might help prevent consequences for people with epilepsy - if there are basic studies that need to be done, data that needs to be gathered, we should do it. Global change is moving ahead. As many 
have said, "There can be no Plan B, because there is no planet B". We cannot fiddle whilst the temperature rises.

Sanjay M Sisodiya, Ingrid E Scheffer, Daniel H Lowenstein, Samantha L Free

Affiliations (I see these seem not to appear in Insight articles, but in case needed):

SMS: Department of Clinical and Experimental Epilepsy, UCL Queen Square Institute of Neurology, London WC1N 3BG, UK

IES: University of Melbourne, Austin Health and Royal Children's Hospital, Florey and Murdoch Institutes, Melbourne, Australia

DHL: Department of Neurology, University of California, San Francisco, San Francisco, CA 94143, USA

SLF: Low Carbon Chilterns Cooperative, UK www.lowcarbonchilterns.org

Corresponding author contact: $\underline{\text { s.sisodiya@ucl.ac.uk }}$

\section{Figure Legend}

The black circles represent the average number of tonnes of carbon dioxide (numerical amount in red numerals) produced per person per year in the given regions (North America, Central America, South America, Africa, Europe, The Middle East, Asia and Oceania). In 2016, the global average amount was 4.8 tonnes of carbon dioxide $\left(\mathrm{tCO}_{2}\right)$ The three rectangles represent one year's additional carbon emissions from work-related air travel (red) for DHL (centred on west coast of USA in map), SMS (centred on UK) and IES (centred on Australia), added to the average annual carbon emissions per person living in those nations (blue).

(Figure taken from www.globalcarbonatlas.org) 